\title{
EL CULREG COMO JUEGO DIDÁCTICO EN EL APRENDIZAJE DE LOS CULTIVOS DE LA REGIÓN, MÉRIDA VENEZUELA
}

THE CULREG AS A TEACHING GAME IN THE LEARNING OF THE CROPS OF THE REGION, MERIDA VENEZUELA

\section{RESUMEN}

El artículo refleja los resultados de una investigación - acción, donde se aplicó un juego para el aprendizaje agrícola evaluando el antes y después; como resultado se obtuvo el diseño e implementación del "Culreg", un juego didáctico en el aprendizaje de los "Cultivos de la Región", ejecutado en una institución Pública de Educación Media General en el estado Mérida, República Bolivariana de Venezuela. La creación del juego didáctico, tuvo como propósitos fundamentales; mejorar el proceso de enseñanza, aprendizaje y facilitar a los docentes alternativas para la enseñanza, en respuesta a la situación que existía, donde los docentes no planifican juegos como estrategia de enseñanza, como una herramienta innovadora en sus actividades académicas. Como resultado se logró enfocar a los jóvenes en un aprendizaje satisfactorio en el proceso de formación agrícola, logrando mayor comprensión, memorización, incremento de la capacidad de análisis de los postulados teóricos y los resultados prácticos y cumplir conscientemente las normas en las actividades planificadas, en los cultivos de Naranja y Lechosa, respecto al proceso de preparación, siembra, conocimiento de plagas, nomenclatura científica y desarrollo de la capacidad de trabajar en grupo, por lo que se recomienda a los docentes desarrollar este tipo de actividades.

Palabras clave: culreg; didáctica; educación; juego.

\section{ABSTRACT}

The research was framed in the qualitative paradigm through research-action, field design, applying assessments to students; before and after the execution of the game in agricultural learning. In this article, teachers do not plan games as a teaching strategy. They do not use it as an innovative tool in their academic activities. In the young people in agricultural training, it focuses on a satisfactory learning, thus achieving to understand, to memorize, to analyze and to repeat the theoretical, practical and to comply with the rules of the game in the planned activities, that is to say, to improve the knowledge in the orange and milky items, to know the process of preparation, planting, scientific names, pests and working in a group. Teachers are encouraged to include and develop such activities and strategies.

Keywords: culreg; didactics; education; game. 


\section{INTRODUCCIÓN}

Dentro del campo educativo es importante vincular los programas de estudio con las actividades didácticas, que formen parte del proceso enseñanza - aprendizaje, ampliando así los nuevos proyectos que exige el perfil para lograr un estudiante, más participativo, reflexivo y protagónico, en las actividades planteadas (Chávez 2007:09).

Ante esta realidad, las Instituciones Educativas se han dado a la tarea de perfeccionar su desempeño en diversos aspectos, uno de ellos el Social, que se debe enfocar al desarrollo personal del adolescente; en las esferas intelectual y afectiva. Es allí, en 1 búsqueda de alternativas para el perfeccionamiento, donde surge la iniciativa de integrar los juegos didácticos al proceso educativo, a fin de promover nuevas formas de enseñar, más activas, incorporando experiencias que tengan un impacto social, donde el estudiante se interese por gestionar el conocimiento, a través de acciones que le permiten proyectar sus potencialidades hacia el medio en el cual se desenvuelve.

Así mismo,

... el juego es uno de esos beneficios, digno de ser apreciado como algo fundamental en el proceso de enseñanza y maduración del individuo, con posibilidades de ser considerado pedagógicamente como un medio y como un fin en sí mismo del desarrollo humano. (Muñoz 2008:23).

En este sentido, surgen las siguientes interrogantes:

- ¿aplican los docentes estrategias que permitan el uso de juegos didácticos?,

- ¿los docentes son innovadores en sus espacios destinados a la educación agrícola?,

- ¿cuál es el interés de los docentes y estudiantes por los juegos didácticos?,

- ¿qué valor tiene el juego como recurso de aprendizaje en los cultivos de la región?
El análisis de las preguntas planteadas conduce a la reflexión sobre el desempeño real de los docentes, pues aunque los mismos tienen la responsabilidad de mejorar su práctica pedagógica con estrategias innovadoras y creativas, en realidad existe un nivel insuficiente de aplicación de estrategias pedagógicas que potencien el uso de juegos didácticos en el proceso de formación e incluso los estudiantes se quejan del excesivo uso de clases magistrales, donde el docente es el que asume un papel activo mientras los alumnos son pasivos receptores, lo que implica, la necesidad de implementar juegos como alternativa para la enseñanza de los jóvenes, asignándoles un rol interactivo en la gestión del conocimiento y favoreciendo un mejor y mayor desarrollo del proceso de enseñanza aprendizaje relacionado con los cultivos de la región, en el Área de Educación para el Trabajo y Desarrollo Endógeno.

Siguiendo el mismo orden de ideas, el propósito es el estudio y el desarrollo del Culreg (Cultivos de la Región), como Juego Didáctico en una institución pública del nivel de Educación Media General, en el Estado Mérida, que se concibe para potenciar la motivación de los estudiantes por adquirir conocimientos teóricos y prácticos y despertar su interés por aprender sobre los Cultivos de la Región y materias afines a la educación, con la ayuda de los docentes, cambiando la planificación docente tradicional, por una más dinámica, alegre y participativa, sin perder el sentido educativo dentro y fuera del aula.

Con el Culreg además, se logrará una participación comprometida en lo individual y grupal, al ejecutar el juego didáctico, mejorando la comprensión, comunicación, análisis, así como el planteamiento interrogantes y la búsqueda de respuestas relacionadas con los cultivos de Naranja y Lechosa y del 
contenido que forma parte de la agricultura educativa en el Área de Educación para el Trabajo.

En tal sentido, el elemento lúdico beneficiará el aprendizaje en los jóvenes, y podrá ser tomado como un recurso importante, ya que brinda considerables ventajas en el proceso de enseñanza - aprendizaje, pudiendo ser utilizado como estrategia expresiva, en tanto relaja y motiva a los estudiantes; como estrategia comunicativa, ya que implica un proceso de comunicación real dentro y fuera del aula; y como estrategia para la fijación de nuevos contenidos, cuando el propósito del juego radica en profundizar sobre la comprensión de las relaciones de dependencia e interacción entre los elementos componentes de un fenómeno o proceso estudiado.

El cambio necesario en el sistema educativo, representa ir más allá de las estrategias aplicadas en el aula de clase, es así que desde la perspectiva de la didáctica, la aplicación de nuevas ideas tiene utilidad para motivar e integrar a los estudiantes en el propósito de retomar nuevos conocimientos y asimilar las teorías y prácticas en un ambiente escolar armónico, lleno de alegría, satisfaciendo sus necesidades, educando mediante el juego. Desde este punto de vista el juego didáctico en el espacio libre-cotidiano es de mucha utilidad educativa, aunque es diferente, al juego dentro de un espacio organizado e institucionalizado, como el liceo.

\section{METODOLOGÍA}

La investigación que sirvió de base al artículo, se enmarcó en el paradigma cualitativo, concebida en la variante de Investigación-Acción, "una opción metodológica que pretende resolver un problema real y concreto, sin ánimo de realizar ninguna generaliza- ción con pretensiones teóricas" (UPEL 2008:36), y donde los mismos actores de los procesos cotidianos de trabajo intervienen en la identificación de problemas y en el proceso de transformación de la realidad. La investigación incluyó además trabajo de campo, en tanto los datos de interés fueron recogidos en forma directa de la realidad por el investigador.

En tal sentido, la investigación cualitativa hace referencia a "la recolección de datos, análisis e interpretación que no son objetivamente mensurables, es decir, que no se pueden sintetizar en forma de números. Sin embargo, esto no implica una falta de objetividad de los resultados" (UPEL 2008:33), que en muchas ocasiones reflejan estados de opinión y percepciones de los actores implicados en el proceso de medición.

La investigación busca plantear soluciones reales a problemáticas que están presentes en el Liceo Bolivariano JJOR, institución pública del estado Mérida, mediante la implementación de un juego didáctico que llene las expectativas de los jóvenes en la enseñanza de los Cultivos de la Región (Culreg), estimulando así su proceso de formación.

Se trabajó directamente con jóvenes del Segundo (2do) Año de Educación Media General, con una matrícula de 17 estudiantes y dos profesores en el área de educación para el trabajo de educación media general, especialistas en desarrollo endógeno, no residentes en la zona y con un tiempo de servicio de 10 años.

Estas personas conformaron el universo de la unidad de análisis que sirvió como estudio de caso (estudio de caso de tipo intencional y único), por lo cual el muestreo aplicado fue de tipo: no probabilístico. Los estudiantes que integraron la muestra presentan las 
siguientes características:

- edades comprendidas de 12 a 14 años,

- residentes de la zona de estudio,

- con un conocimiento previo sobre los cultivos de la región.

Se empleó una guía de observación estructurada que se diseñó con el fin de diagnosticar la planificación diaria de los docente en el aula, qué actividades desarrollan sobre la enseñanza, aprendizaje del tema "Cultivos de la Región" y la aplicación de juegos para la enseñanza de la agricultura, además de identificar los conocimientos de los estudiantes y docentes de educación media general sobre los cultivos de la región y los juegos didácticos.

\section{RESULTADO Y DISCUSIÓN}

Es importante integrar los juegos en el proceso enseñanza - aprendizaje, logrando así, jóvenes motivados e interesados por aprender sobre los cultivos de la región del estado Mérida Venezuela. Este principio sirvió de inspiración a la investigación desarrollada, donde en cada una de las etapas se obtuvieron los resultados siguientes:

\section{Planificación del Juego Didáctico Culreg "Cultivos de la Región"}

El desarrollo del juego didáctico para el aprendizaje de los cultivos de la región "Culreg" nos permite facilitar la unificación de los contenidos en los cultivos de naranja y lechosa. Contribuir a la elección de estrategias y recursos para la enseñanza, así como, acciones que permitan una adecuada intervención didáctica en el aula. Incentivar a los estudiantes de educación media general por los juegos, llenando las expectativas de los educandos en la participación individual o agrupada al momento de poner en práctica este tipo de Juego Didáctico.

En este sentido, el juego proporciona en los educandos nuevas representaciones para explorar la realidad y estrategias diferentes para aplicar sobre ésta, favoreciendo un lugar para lo creativo, en el mundo que lo rodea, donde en su totalidad las cosas ya están creadas, permiten a los jóvenes, expresar nuevas espacios de su imaginación, haciéndolo pensar en numerosas alternativas para solucionar una problemática y que favorecen el cambio de conducta durante su proceso de enseñanza, aprendizaje. (García 2006:23)

En este sentido, se utilizó una planificación didáctica orientada por el docente especialista del área agrícola, apoyada en actividades de inicio, desarrollo y cierre. Utilizando como material reciclable como soporte logístico o de apoyo: recortes de tablas, martillo, clavos, cartón, hojas blancas, tarjetas telefónicas, goma, tijeras, reglas, lápiz, computadora, láminas para plastificados.

En la figura 1, se puede apreciar a los estudiantes trabajando con el material de referencia.

\section{Inicio de la Actividad}

La actividad de aplicación del Juego Didáctico Culreg, fue iniciada por el profesor, con la socialización de los objetivos de la actividad, luego preguntas por parte de los estudiantes y posteriormente lluvia de ideas (brainstorming), dirigida por el docente durante la elaboración del juego. Finalmente se aplicó una prueba escrita (previa al desarrollo del juego didáctico), sobre los contenidos de los cultivos de naranja y lechosa, para tener tomar las respuestas como puno de partida y fuente de comparación posterior sobre el aprendizaje adquirido por los jóvenes.

Es importante tomar en cuenta que los jóvenes en formación tienen un conocimiento previo antes de aplicar este tipo de Juego Didáctico; ya que viven y se desarrollan en una zona rural, en este caso referente al estado Mérida, República Bolivariana de Venezuela. 


\section{Unach}

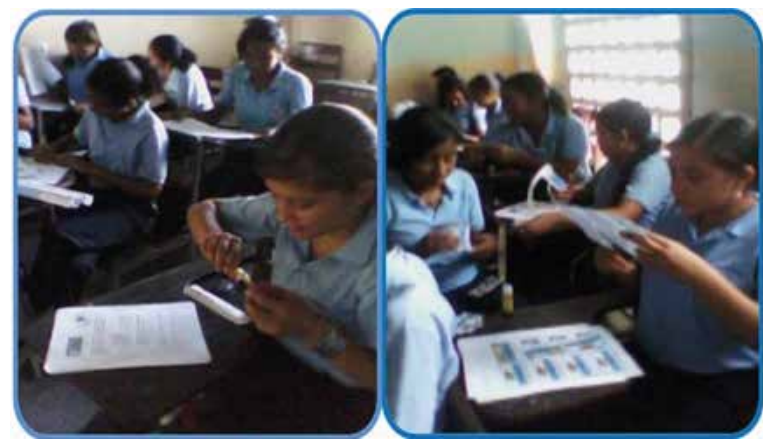

Figura 1: Material utilizado en el Juego Didáctico Fuente: elaboración propia.

No obstante, tal y como se refleja en la tabla 1 los resultados de la prueba escrita aplicada a los jóvenes del 2do año de educación media general antes de poner en práctica el "Culreg", expresan desconocimiento sobre los cultivos de naranja citrus sinensis $\mathrm{y}$ lechosa carica papaya, pues un análisis porcentual de respuestas por preguntas refleja rangos de respuestas incorrectas que fluctúan entre $41 \%$ y $94 \%$, por otra parte, entre el $6 \%$ y $94 \%$ de los estudiantes no responden algunas preguntas.

Tabla 1: Resultados de la prueba escrita antes de la aplicación del Juego Didáctico. Datos tomados de la prueba escrita antes del desarrollo del Juego

\begin{tabular}{|c|c|c|c|c|}
\hline PatcinatA & Antes & c & in & MR \\
\hline 1;:0et in in Agicolturn? & & 0 & $94 \%$ & $6 \%$ \\
\hline 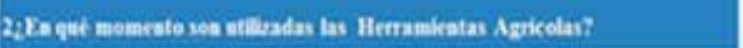 & & $24 \%$ & $76 \%$ & 0 \\
\hline 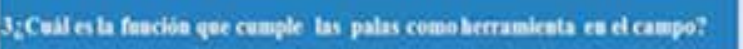 & & 646 & $82 \%$ & $12 \%$ \\
\hline 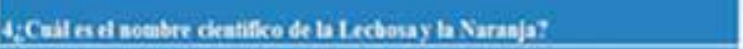 & & 0 & $71 \%$ & $29 \%$ \\
\hline PREGENA & Antes & c & I & NR \\
\hline 58Call e la aluma Promedis de eas planta de Lechosay de Narneja? & & 0 & $47 \%$ & $53 \%$ \\
\hline 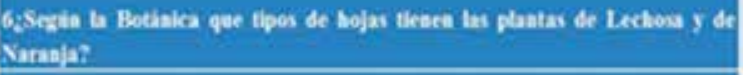 & & 0 & 64 & $94 \%$ \\
\hline 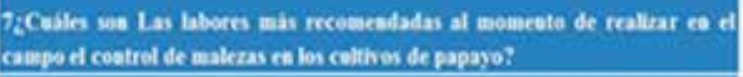 & & $18 \%$ & $76 \%$ & $6 \%$ \\
\hline 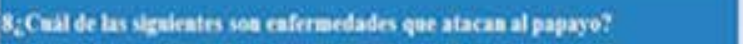 & & $18 \%$ & $64 \%$ & $18 \%$ \\
\hline PREGENTA & Antes & c & I & N.R \\
\hline 92006 mitodevertitin pare recelectar lo papea? & & $59 \%$ & $41 \%$ & 0 \\
\hline 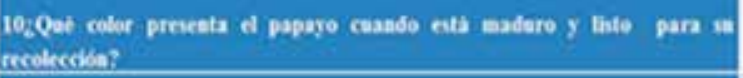 & & $35 \%$ & $59 \%$ & $6 \%$ \\
\hline $\begin{array}{l}\text { 118Q0e ther de podas re realiran ea las pricticas de campo ea el Caltho de } \\
\text { Naranja? }\end{array}$ & & $47 \% 6$ & $47 \%$ & $6 \%$ \\
\hline
\end{tabular}

Fuente: Elaboración propia a partir de la prueba efectuada.

LEYENDA:

$\mathbf{C}=$ Correctas $=$ Especifica la cantidad de preguntas respondidas correctamente.

I $=$ Incorrectas $=$ Detalla la cantidad de preguntas respondidas incorrectamente.

$\mathbf{N R}=$ No respondidas $=$ Describe la cantidad de preguntas no respondidas. 


\section{Desarrollo del Juego Didáctico}

En esta etapa se diseñó el juego con las orientaciones del docente e integración de los educandos. Seguidamente se organizaron dúos de estudiantes para desarrollar el Juego Didáctico, como parte del proceso de enseñanza - aprendizaje. En la figura 2, se pueden apreciar los jóvenes estudiantes en el momento en que juegan.

Durante el desarrollo del Culreg, los investigadores aplicaron la observación como técnica para constatar el comportamiento de los estudiantes respecto al aprovechamiento de las potencialidades del Juego Didáctico ejecutado, su efecto en el conocimiento de las particularidades de los cultivos de naranja y lechosa, los aspectos más significativos, derivados de la conducta y el desempeño seguido por los alumnos, fueron registrados convenientemente.

\section{Cierre de la Actividad}

Como parte conclusiva o etapa de cierre, se efectuó una prueba escrita, luego de aplicado el Juego Didáctico Culreg, con la finalidad de evaluar los conocimientos adquiridos sobre los cultivos de naranja y lechosa característicos de la región, llegando a deter- minar la gran importancia que para los estudiantes del área agrícola tiene la utilización de este Juego Didáctico para reforzar los conocimientos.

En la figura 3, se puede apreciar a algunos estudiantes durante la etapa de cierre.

Un análisis de las respuestas aportadas por los estudiantes del 2do año de educación media general, en la prueba efectuada después de aplicar el juego didáctico Culreg, se aprecian en la tabla 2, donde se pudo comprobar que este tipo de actividad mejora el entendimiento de los temas aplicados en el juego.

En tal sentido es significativo que el análisis porcentual de las respuestas correctas por pregunta, presenta un rango que fluctúa entre el $94 \%$ y $100 \%$, valores significativamente inferiores a los porcentajes de respuestas incorrectas y no respondidas antes de aplicar el "Culreg".

Por otra parte fue notable el avance progresivo en la capacidad de memorización, comunicación, análisis, asimilación de contenidos y métodos que se practican en el campo agrí-

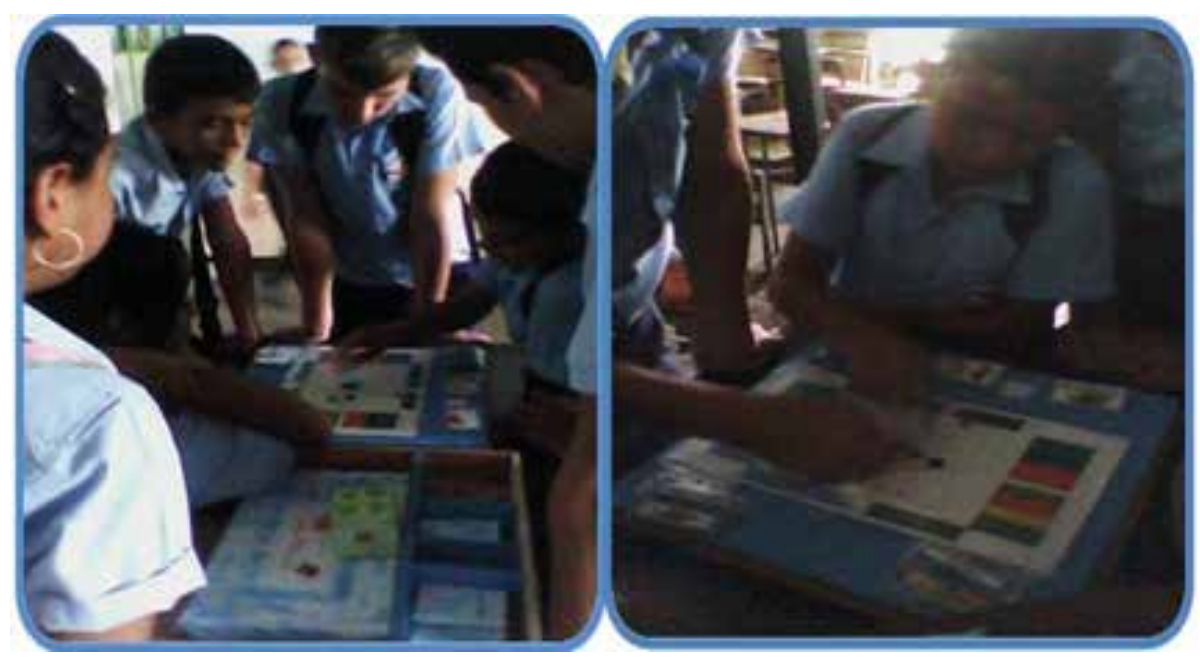

Figura 2: Desarrollo del Juego Didáctico

Fuente: elaboración propia.

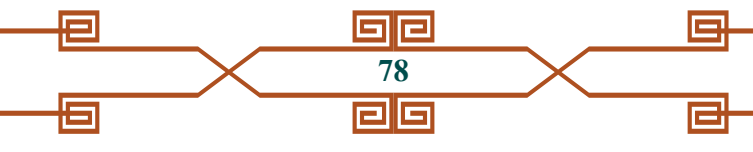




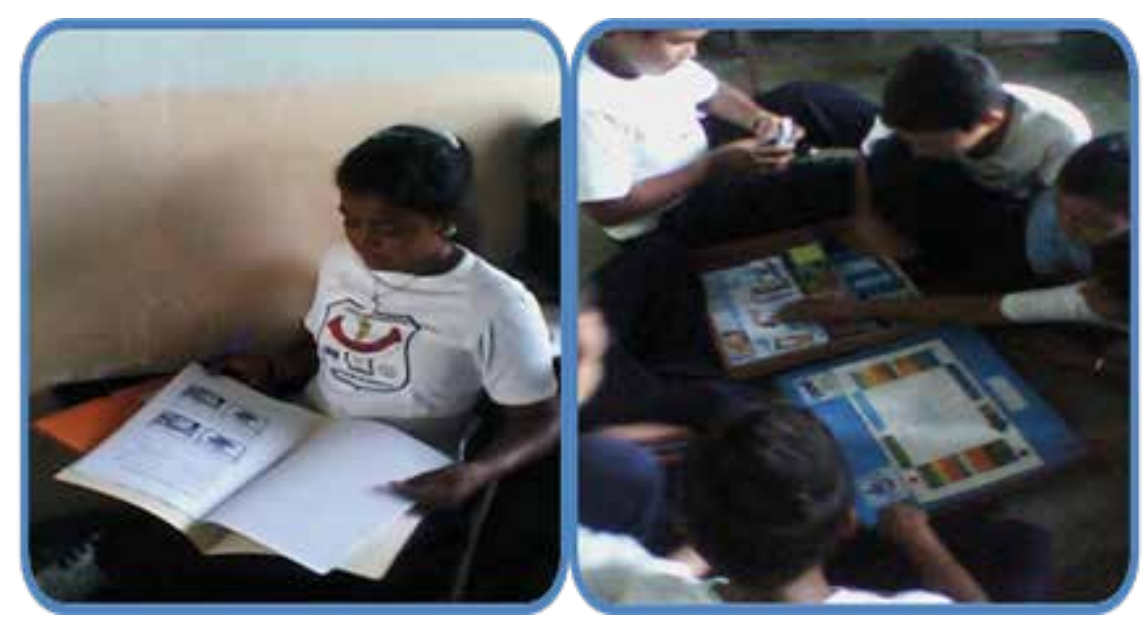

Figura 3: Cierre de la actividad y Evaluación aplicada Fuente: elaboración propia.

cola y que son puestos en práctica en los campos de cultivo de la naranja y la lechosa, tales como: forma de recolectar los frutos, enfermedades que afrontan los cultivos, tipos de hojas de las plantas, condiciones hídricas, la función y utilización de las herramientas.

Lo anterior, la participación de los jóvenes en el diseño y desarrollo del "Culreg", les permitió conocer nuevas alternativas para su formación académica y se observó el aumento de interés de los jóvenes durante la actividad, lo que ayudó al buen desempeño en la discusión grupal y análisis individual del contenido programado en la planificación.

En este sentido, el juego es una acción que a través de los jóvenes se realiza en forma espontánea, que desarrolla integralmente la personalidad de los educandos y en particular su capacidad de poder crear, donde el intelectual cognitivo se fomente en la observación, las capacidades lógicas, la ilusión, la decisión, las instrucciones, las habilidades y el potencial para crear en los educandos nuevas alternativas para solucionar los problemas presentes.

Como complemento informativo y fuente de análisis, en la tabla 3 se expresan los resulta- dos obtenidos en la prueba antes y después de la aplicación del Juego Didáctico Culreg, donde producto de la comparación resulta evidente un nivel más amplio de los conocimientos relacionados a los cultivos de la región, naranja y lechosa, en la formación del grupo de jóvenes que integraron el caso de estudio, donde se implementó este juego didáctico, lo que se manifiesta en altos porcentajes.

Entre otros aspectos se evidenció; el "Culreg" ayuda a fortalecer la enseñanza aprendizaje en el tema de estudio, permitiendo una participación activa de los educandos en el diseño y desarrollo del juego. Así mismo, el buen desempeño en la actividad, siendo más organizada; ya que se cumplen las normativas establecidas para el buen desenvolviendo de las acciones planteadas.

En este sentido, el juego es una acción, por medio de los jóvenes; que se realiza en forma espontánea, que desarrolla integralmente la personalidad de los educandos, y en particular su capacidad de poder crear. Donde se fomentan: la observación, las capacidades lógicas, las habilidades, el potencial para crear, y la preparación de los educandos en alternativas para solucionar los problemas presentes. 
Tabla 2: Resultados de la prueba escrita después de la aplicación del Juego Didáctico, datos tomados de la prueba escrita después del desarrollo del Juego

\begin{tabular}{|c|c|c|c|c|}
\hline PREGEIA & Derash. & C & 1 & $N R_{2}$ \\
\hline 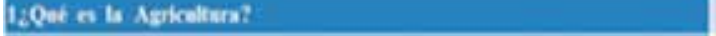 & & $88 \%$ & 1246 & 0 \\
\hline 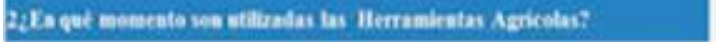 & & $100 \%$ & 0 & 0 \\
\hline 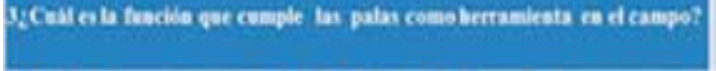 & & 945 & $6 \%$ & 0 \\
\hline 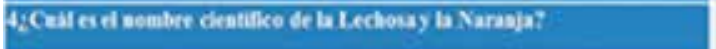 & & 1006 & 0 & 0 \\
\hline PREGINA & Despots & c & 1 & NR \\
\hline 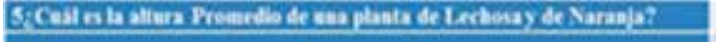 & & 946 & e & os \\
\hline 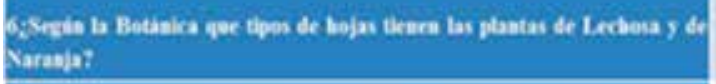 & & 1006 & 0 & 0 \\
\hline 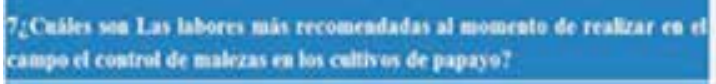 & & 945 & 60 & 0 \\
\hline 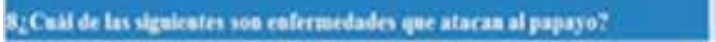 & & 946 & 606 & 0 \\
\hline PREGINIA & Despols & c & 1 & N.R \\
\hline 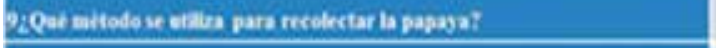 & & $100 \%$ & 0 & 0 \\
\hline 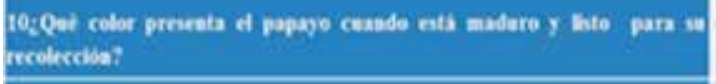 & & $100 \%$ & 0 & 0 \\
\hline 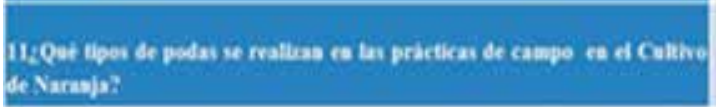 & & $100 \%$ & 0 & 0 \\
\hline
\end{tabular}

Fuente: elaboración propia a partir de la prueba efectuada.

LEYENDA:

$\mathbf{C}=$ Correctas $=$ Especifica la cantidad de preguntas respondidas correctamente $\mathbf{I}=$ Incorrectas $=$ Detalla la cantidad de preguntas respondidas incorrectamente $\mathbf{N R}=$ No respondidas $=$ Describe la cantidad de preguntas no respondidas

Tabla 3: Comparación del Aprendizaje en los Cultivos de la Región, datos tomados de la prueba escrita antes y posterior a la aplicación del Juego Didáctico "Culreg"

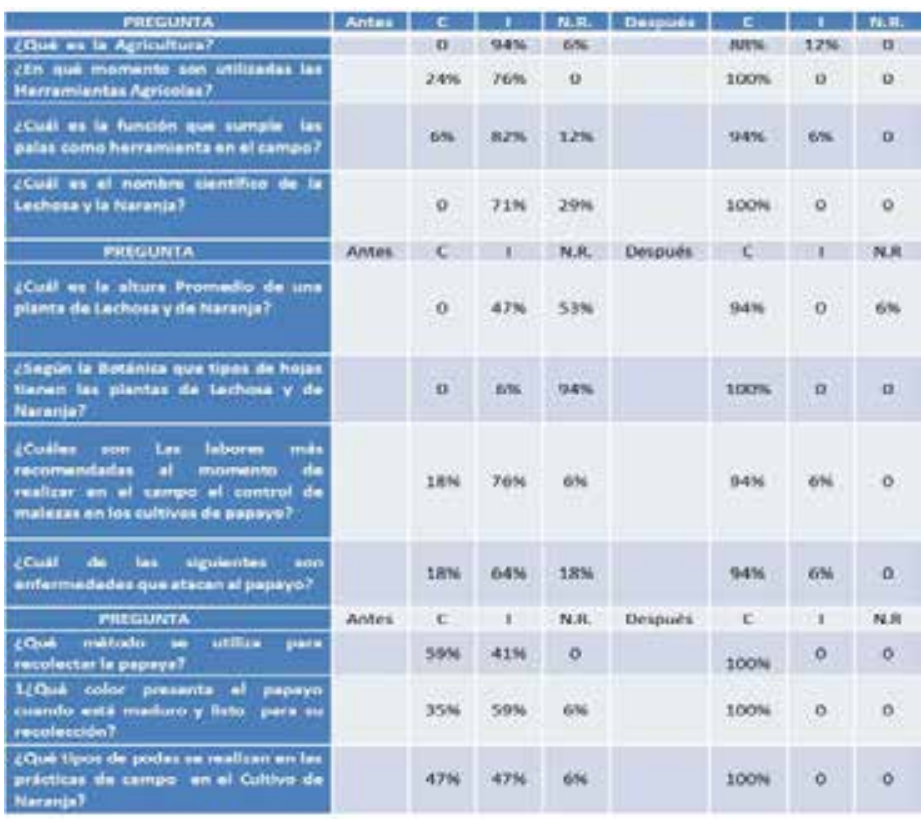

Fuente: elaboración propia a partir de la prueba efectuada.

回


LEYENDA:

$\mathbf{C}=$ Preguntas correctas

$\mathbf{I}=$ Preguntas incorrectas

$\mathbf{N} . \mathbf{R}=$ Preguntas no respondidas

Esto podría constituirse en elemento facilitador para cumplir con lo que se establece en las bases legales de la Constitución de la República Bolivariana de Venezuela (2000), en su artículo $3^{\circ}$, en la Ley Orgánica de Protección al Niño y al Adolescente (1998), en su artículo 58 (LOPNA) y la Ley Orgánica de Educación (2009), en su artículo 14, que preconizan la necesidad de armonizar la educación con las actividades productivas propias del desarrollo social, local, regional y nacional a través de la orientación de niños, niñas, adolescentes y jóvenes; formándolos(as) en, por y para el trabajo liberador, creador, innovador y productivo con visión significativa de lo humano, que permita satisfacer necesidades básicas, contribuir al desarrollo regional y nacional.

\section{CONCLUSIONES}

La elaboración e implementación del juego didáctico "Culreg" como una estrategia para mejorar el proceso de enseñanza - aprendizaje relacionada con los cultivos de naranja citrus sinensis y lechosa carica papaya, característicos de la región, contribuyó a fomentar el interés de los estudiantes, su motivación individual y grupal, lo que quedó evidenciado los resultados alcanzados por los estudiantes cuando se les aplicó la prueba luego de la aplicación del "Culreg", donde el análisis porcentual reflejó entre el $94 \%$ y el $100 \%$ de respuestas correctas a cada pregunta, muy superior al resultado obtenido en la prueba efectuada antes del "Culreg", que reflejó entre el 41\% y el 94\%, de respuestas incorrectas por pregunta, unido a que hubo entre un $6 \%$ y un $94 \%$ de estudiantes que no respondieron alguna de las preguntas formuladas.

Para finalizar, el juego didáctico en lo académico; constituyó una alternativa para los docentes relacionada con la planificación y ejecución del proceso formativo, tanto en las actividades teóricas, como prácticas.

\section{RECOMENDACIONES}

Incorporar los juegos didácticos como el "Culreg" en la matriz curricular de la institución e instituciones educativas de localidades agrícolas, para fortalecer los conocimientos vinculados a los cultivos del área, lo que además puede constituirse en puente integrador de las diferentes áreas del aprendizaje como la matemática, física, química y la lengua materna, entre otras.

Tomar en cuenta las necesidades de los educandos al momento de hacer la planificación didáctica, para diversificar las formas organizativas de docencia y llevar a cabo actividades recreativas, innovadoras, interesantes, que cambien la conducta de los jóvenes, sin perder el sentido educativo.

Organizar en el aula de clase espacios predestinados a los juegos didácticos en beneficio de los estudiantes y adaptar los textos escolares las nuevas exigencias del sistema educativo nacional e internacional.

Fomentar el intercambio metodológico y la reflexión entre los docentes, propiciando la debida organización de actividades didácticas, apoyadas en la planificación pedagogía para mejorar la enseñanza sobre los cultivos de la región y otros contenidos de la educación.

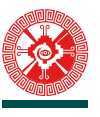




\section{Unach}

\section{REFERENCIAS BIBLIOGRÁFICAS}

Constitución de la República Bolivariana de Venezuela. (1999). Caracas, Venezuela.

Chávez, A. (2007). Currículo Nacional Bolivariano. Caracas, Venezuela: Fundación Centro Nacional para el Mejoramiento de la Enseñanza de Ciencia.

Información Técnica Agrícola. (2013). Curso de Citricultura. Recuperado de http://www.infoagro.com/citricos/naranja.htm

García, A. (2006). El juego. La clasificación de los Juegos. Otros Tipos de Juegos comunes en la Primera Infancia. Recuperado de http://www.waece.org/enciclopedia/resultado2.php?id=10110

Información Técnica Agrícola. (2013). Fruticultura subtropical. Recuperado de http://www.infoagro.com/frutas/frutas.htm

Ley Orgánica de Educación. Publicada en La Gaceta Oficial No. 5929E, del 15 de agosto del 2009. Venezuela.

Ley Orgánica de Protección al Niño y al Adolescente. Publicada en La Gaceta Oficial No. 5.266, del 2 de octubre de 1998. Venezuela.

Muñoz, A. (2008). Educar en valores y Aprender Jugando. España: Editorial MAD, S.L.

UPEL, (2008). Investigación Educativa. Caracas, Venezuela: Universidad Pedagógica Experimental Libertador.

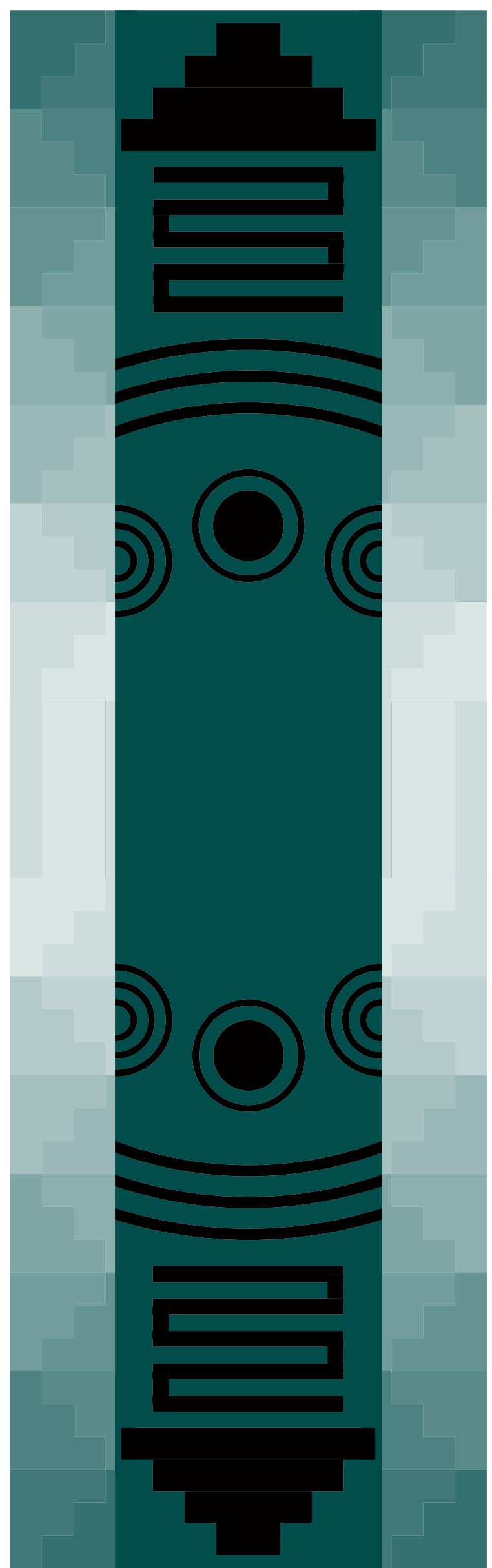

